\title{
Usefulness and pitfalls of MAA SPECT/CT in identifying digestive extrahepatic uptake when planning liver radioembolization
}

\author{
Laurence Lenoir • Julien Edeline • Yann Rolland • \\ Marc Pracht • Jean-Luc Raoul • Valérie Ardisson • \\ Patrick Bourguet • Bruno Clément • Eveline Boucher • \\ Etienne Garin
}

Received: 23 September 2011 / Accepted: 8 December 2011 /Published online: 12 January 2012

(C) Springer-Verlag 2012

\begin{abstract}
Purpose Identifying gastroduodenal uptake of ${ }^{99 \mathrm{~m}} \mathrm{Tc}$ macroaggregated albumin (MAA), which is associated with an increased risk of ulcer disease, is a crucial part of the therapeutic management of patients undergoing radioembolization for liver tumours. Given this context, the use of MAA single photon emission computed tomography (SPECT)/CT may be essential, but the procedure has still not been thoroughly evaluated. The aim of this retrospective study was to determine the effectiveness of MAA SPECT/CT
\end{abstract}

\author{
L. Lenoir $\cdot$ V. Ardisson $\cdot$ P. Bourguet $\cdot$ E. Garin $(\bowtie)$ \\ Department of Nuclear Medicine, \\ Comprehensive Cancer Institute Eugène Marquis, \\ CS 44229, 35042 Rennes, France \\ e-mail: etienne.garin@univ-rennes1.fr \\ L. Lenoir $\cdot$ J. Edeline $\cdot$ P. Bourguet $\cdot$ E. Garin \\ University of Rennes 1 , \\ 35043 Rennes, France \\ L. Lenoir · B. Clément · E. Boucher · E. Garin \\ INSERM, U-991, Liver Metabolisms and Cancer, \\ 35033 Rennes, France \\ J. Edeline $\cdot$ M. Pracht $\cdot$ E. Boucher \\ Department of Medical Oncology, \\ Comprehensive Cancer Institute Eugène Marquis, \\ CS 44229, 35042 Rennes, France \\ Y. Rolland \\ Department of Medical Imaging, \\ Comprehensive Cancer Institute Eugène Marquis, \\ CS 44229, 35042 Rennes, France \\ J.-L. Raoul \\ Department of Medical Oncology, \\ Comprehensive Cancer Institute Paoli Calmette, \\ 13273 Marseille, France

in identifying digestive extrahepatic uptake, while determining potential diagnostic pitfalls.

Methods Overall, 139 MAA SPECT/CT scans were performed on 103 patients with different hepatic tumour types. Patients were followed up for at least 6 months according to standard requirements.

Results Digestive, or digestive-like, uptake other than free pertechnetate was identified in $5.7 \%$ of cases using planar imaging and in $36.6 \%$ of cases using SPECT/CT. Uptake sites identified by SPECT/CT included the gastroduodenal region (3.6\%), gall bladder (12.2\%), portal vein thrombosis $(6.5 \%)$, hepatic artery $(6.5 \%)$, coil embolization site $(2.1 \%)$ as well as falciform artery (5.0\%). For $2.1 \%$ of explorations, a coregistration error between SPECT and CT imaging could have led to a false diagnosis by erroneously attributing an uptake site to the stomach or gall bladder, when the uptake actually occurred in the liver.

Conclusion SPECT/CT is more efficacious than planar imaging in identifying digestive extrahepatic uptake sites, with extrahepatic uptake observed in one third of scans using the former procedure. However, more than half of the uptake sites in our study were vascular in nature, without therapeutic implications. The risk of coregistration errors must also be kept in mind.

Keywords Radioembolization · SPECT/CT · MAA

\section{Introduction}

Radioembolization based on microspheres labelled with ${ }^{90} \mathrm{Y}$ has emerged as a valuable therapy that is increasingly being used for patients with liver tumours [1-3]. In contrast to ${ }^{131}$ I-lipiodol therapy, this therapeutic approach has not only shown efficacy in patients with hepatic carcinomas, but the 
procedure may also be used for treating hepatic metastases [4, 5]. In addition to its efficacy and association with fewer constraints in terms of radiation protection, another distinct advantage of the technique is its low toxicity profile. In addition, this therapeutic strategy is associated with a low risk of pulmonary complications or gastroduodenal ulcer disease when these structures are irradiated. Therefore, due to safety concerns, this technique requires an initial diagnostic angiography followed by hepatic arterial perfusion scintigraphy using ${ }^{99 \mathrm{~m}} \mathrm{Tc}$-labelled albumin macroaggregates (MAA) in order to identify potential pulmonary or digestive shunts.

While the technique has been primarily used to identify pulmonary shunts, with the development of single photon emission computed tomography (SPECT)/CT imaging, MAA scintigraphy is now increasingly being used to detect digestive shunts, which may constitute a contraindication to this type of therapy. Recently, a number of studies revealed the usefulness of SPECT/CT in identifying gastroduodenal uptake sites, notably in cases where no arterial shunts had been detected on angiography [6-9]. Nonetheless, MAA SPECT/CT semiology has not been fully established.

The aim of this retrospective study was to determine the usefulness of MAA SPECT/CT in identifying digestive extrahepatic uptake sites, corresponding to digestive shunts that were likely to alter the therapeutic planning, while determining potential diagnostic pitfalls.

\section{Materials and methods}

Data from 139 MAA SPECT/CT scintigraphic explorations involving 103 consecutive patients (79 men and 24 women) undergoing ${ }^{90} \mathrm{Y}$-microsphere therapy were analysed retrospectively. The choice of selective internal radiation therapy (SIRT) was taken by a multidisciplinary team focused on liver cancer management. Regarding tumour types, 78 patients suffered from hepatocellular carcinoma (HCC), 13 from cholangiocarcinoma and 12 from hepatic metastases (neuroendocrine, $n=7$; colorectal, $n=3$; choroid melanoma, $n=2$ ). Written informed consent was obtained from each patient. Prior to microsphere therapy, primary diagnostic selective hepatic angiography was performed in all patients, along with coil embolization of digestive arteries as appropriate, which was followed by MAA scintigraphy. In 89 patients, ${ }^{90}$ Y-microsphere therapy was conducted (glass microsphere, $n=84$; resin microspheres, $n=6$ ), the treatment being contraindicated in 14 patients due to excessive pulmonary shunting in 5 , non-correctable digestive shunting in 2, insufficient tumour targeting in 6 and hepatic deterioration occurring between diagnostic angiography and therapy initiation in the remaining patient.

After selectively injecting $185 \mathrm{MBq}$ MAA into the right or left hepatic artery, MAA scintigraphy was performed using planar imaging $(256 \times 256,5 \mathrm{~min})$ and SPECT/CT $(32$ projections, $128 \times 128,180^{\circ}$ and $30 \mathrm{~s} /$ frame). SPECT data were reconstructed with iterative methods (ordered subset expectation maximization, 5 iterations, 8 subsets), following attenuation and diffusion correction, and then fused with CT data using a Syngo data-processing console display unit (Siemens, Erlangen, Germany). After the indication was confirmed, ${ }^{90} \mathrm{Y}$ microsphere therapy was carried out within 15 days after the first diagnostic angiography. Post-therapeutic bremsstrahlung scintigraphy (BS) was carried out (windows $70 \pm 15 \%, 135 \pm$ $15 \%$, nd $167 \pm 15 \% \mathrm{keV})$ using both planar imaging $(256 \times 256$, $5 \mathrm{~min}$ ) and SPECT/CT (32 projections, $128 \times 128,180^{\circ}$ and $30 \mathrm{~s} /$ frame).

Planar images and SPECT/CT data were reviewed independently by two nuclear medicine specialists experienced in radioembolization for liver tumours. In the case of disagreement, a consensus was reached.

\section{Patient follow-up}

Patients were followed up at 6 weeks and 3 months, and then in 3-month intervals in order to assess clinical and biological tolerance, as well as responses using contrast-enhanced CT. In the case of digestive complaints, a gastroduodenal fibroscopy was performed. Only patients with at least 6 months of followup were included in the final analysis.

\section{Results}

Digestive MAA uptake or extrahepatic digestive-like uptake was noted in $81 \mathrm{SPECT/CT}$ scans (Table 1), comprising 31 cases of gastric uptake due to free pertechnetate, as shown by diffuse, low to moderately intense gastric uptake, associated with thyroid gland uptake. Therefore, digestive or digestivelike uptake with a potential impact on patient management was observed in 50 SPECT/CT scans, corresponding to $35.9 \%$ of the imaging procedures performed. Data relating to planar scintigraphy and SPECT/CT as regards the identification of abdominal extrahepatic uptake other than pertechnetate are provided in Table 1.

Table 1 Site and number of extrahepatic uptake detected using planar imaging and SPECT/CT

\begin{tabular}{lll}
\hline & Planar acquisition & SPECT/CT \\
\hline Free pertechnetate & 30 & 31 \\
Focal gastroduodenal uptake & 0 & 5 \\
Portal vein thrombosis & 0 & 9 \\
Hepatic artery & 0 & 9 \\
Coil & 0 & 3 \\
Gall bladder & 2 & 17 \\
Falciform artery & 6 & 7 \\
\hline
\end{tabular}


In five patients, there was gastric $(n=2)$ or duodenal $(n=3)$ (Fig. 1) uptake other than free pertechnetate, as defined by focal and isolated uptake with no concomitant thyroid gland uptake, corresponding to $4.8 \%$ of patients. Among these patients, a new angiography permitted the identification of an aberrant artery in the digestive tract of three patients, who underwent successful coil embolization prior to microsphere therapy. Concerning the remaining two patients, re-reading the diagnostic angiograms did not reveal any artery likely to vascularize the stomach or duodenum, and treatment was also contraindicated due to excessive lung shunting in one case and poor targeting in another.

In total, $21 \mathrm{SPECT} / \mathrm{CT}$ scans revealed digestive-like uptake in the area of the hepatic hilum, which after a comparison with contrast-enhanced $\mathrm{CT}$ was attributed to uptake by the vascular structures. Uptake was due to main portal vein thrombosis in nine patients (Fig. 2) and hepatic artery in nine other patients (Fig. 3), while in three patients, uptake was linked to an accessory gastric artery, which was treated using coil embolization (Fig. 4). In 18 patients, radioembolization therapy was performed, with none of the patients presenting digestive complications, although it was contraindicated in one case of excessive pulmonary shunting and in two others with poor targeting. For the eight patients with portal vein thrombosis uptake who underwent radioembolization treatment, microsphere uptake was also found on BS. Among the nine patients with arterial MAA uptake who underwent microsphere therapy, eight did not present any uptake on BS (Fig. 3), whereas in the remaining patient, arterial microsphere uptake was found on BS. Only one of the three patients with MAA uptake at the coil site showed uptake on BS. None of the 18 patients undergoing microsphere treatment experienced digestive complications.

Overall, 17 patients presented gall bladder uptake, which was considered intense in 7 cases, as defined by an uptake intensity superior to that of healthy hepatic parenchyma, and weak in 10 cases. For the seven patients with intense uptake, the catheter could not be placed more distally, while stimulation using the cystic artery catheter was possible in two cases prior to microsphere treatment. BS revealed microsphere uptake in only two cases, one of which showed uptake following cystic artery stimulation. There was no uptake in 15 cases, including 4 which did not undergo cystic artery stimulation (Fig. 5). All of the patients presenting MAA uptake by the gall bladder underwent microsphere treatment and received a 2-week course of corticosteroids and antibiotics after the injection.

Six patients presented moderate to intense uptake in the hepatic falciform artery, which was easily recognizable on planar imaging by its linear trajectory directed towards the umbilicus. For a seventh patient with umbilical hernia, uptake by the anterior abdominal wall was eventually attributed to the hepatic falciform artery, but only after performing SPECT/CT. For six of the seven patients, microsphere treatment was performed without prior hepatic artery embolization, whereas treatment was carried out in the remaining patient following coil embolization of the hepatic falciform artery. None of the seven patients experienced abdominal pain following microsphere treatment.

Lastly, in three cases, SPECT/CT interpretation was rendered difficult, due to misregistration artefacts, possibly suggesting gastric uptake in two cases (Fig. 6) and gall bladder uptake in one. These three patients were treated as a result of the identification of the misregistration artefacts when the MAA SPECT/ CT was performed and not when the retrospective data were analysed. No gastric or biliary complications were observed.

\section{Digestive complications}

Following microsphere therapy, one patient suffered from gastric ulcer disease, which necessitated surgical intervention. This case has already been described [10], with the patient requiring, immediately prior to the microsphere injection, additional coil embolization of the left gastric artery, as gastric MAA uptake was shown on SPECT/CT. The procedure was complicated by distal coil migration, requiring the insertion of a second coil located more proximally to the distant coil. The exact aetiology of the patient's ulcer could not be established, with possible

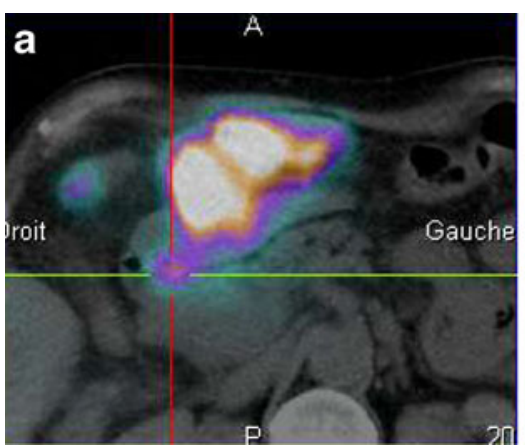

Fig. 1 Uptake at the liver hilum-duodenal uptake. A 69-year-old patient with hepatocellular carcinoma infiltrating the left liver lobe. SPECT/CT revealed focal MAA uptake at the liver hilum, corresponding to a duodenal localization (a), which was not detected on planar imaging. The

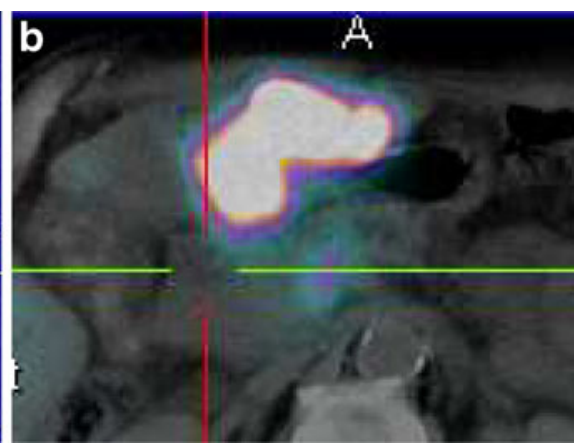

patient underwent a second angiography, which allowed identification of a small supraduodenal artery branch that was successfully treated by coil embolization. After performing a second MAA SPECT/CT, duodenal uptake was no longer apparent (b) 


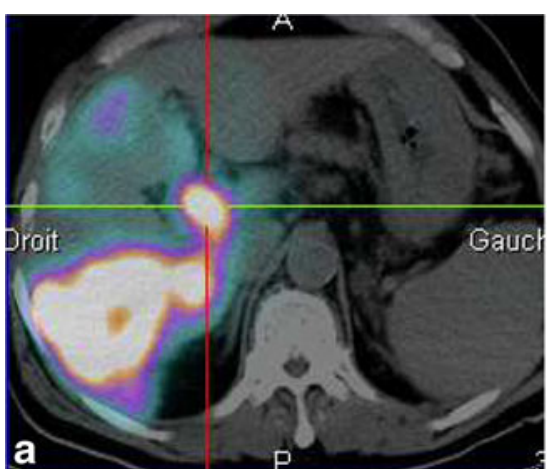

Fig. 2 Uptake at the liver hilum-portal vein thrombosis. Hepatocellular carcinoma patient with main portal vein thrombosis. SPECT/CT revealed focal MAA uptake at the liver hilum (a), corresponding to uptake at the

causes being proximal and distal occlusion of the left gastric artery resulting in acute gastric ischaemia or local irradiation relating to the tumour.

Another patient undergoing treatment with ${ }^{90} \mathrm{Y}$-loaded resin microspheres presented diffuse erosive gastritis, which occurred during a hypertensive crisis requiring the assistance of an anaesthetist. This patient presented a history of treated arterial hypertension, with a recurrence of unifocal HCC on cirrhosis (Child-Pugh score A5). For this patient, neither diffuse nor focal gastric uptake was seen on MAA SPECT/CT, whereas BS did reveal diffuse gastric uptake of mild intensity, suggestive of microsphere reflux occurring during the injection.

Two patients complained of gall bladder pain, which was diagnosed as alithiasic cholecystitis, with sludge features found on ultrasound examination. Both patients' symptoms were resolved following medical treatment. In the first case, MAA SPECT/CT revealed intense gall bladder uptake, which was not observed on BS (Fig. 5). As for the second case, neither MAA SPECT/CT nor BS revealed gall bladder uptake.

\section{Discussion}

Microsphere deposits in the gastrointestinal tract, resulting in an increased risk of developing gastrointestinal ulcer

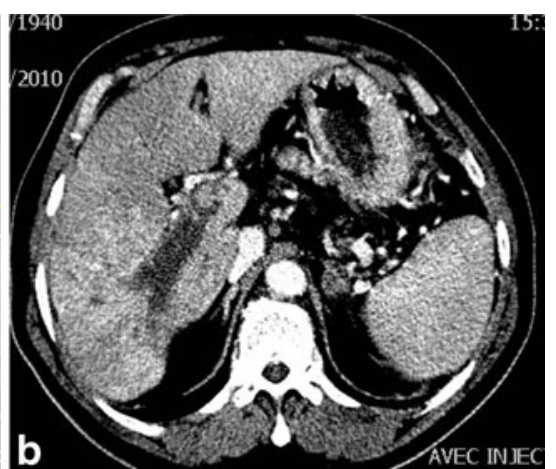

level of the thrombosis clearly seen on the contrast-enhanced CT (b, portal phase). The patient was successfully treated with an objective response with portal vein reperfusion without any complications

disease, are one of the rare complications associated with radioembolization therapy. A recent review of the published literature indicated that the incidence of gastrointestinal ulcers following radioembolization usually ranged from 2.9 to $4.8 \%$ [11], sometimes exceeding $10 \%$, and even approaching 28\% [12-14] in certain series with limited patient numbers. The two major causes thought to be responsible for gastroduodenal ulcers are: (1) digestive shunting via an aberrant gastroduodenal vessel that is subjected to microsphere injection and (2) microsphere reflux occurring during the injection.

Diagnostic angiography is routinely carried out in order to detect aberrant collateral gastrointestinal vessels, while coil embolization is performed in cases where aberrant vessels are disclosed. The disparities in the reported frequencies may be accounted for by several factors: individual case complexities, arterial network alterations due to prior medical or surgical interventions and the different levels of experience of the interventional radiologists (learning curve). In addition, the type of microspheres used must also be taken into account, especially regarding the risk of microsphere reflux during administration. From a theoretical point of view, this risk is probably increased when using resin microspheres rather than glass microspheres, due to their lower specific activity (50 vs $2,500 \mathrm{~Bq} / \mathrm{sphere}$ ) and a

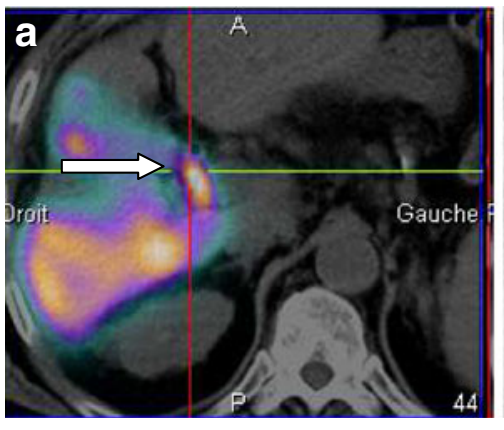

Fig. 3 Uptake at the liver hilum-hepatic artery. A 76-year-old patient with bifocal hepatocellular carcinoma of the right liver lobe. MAA SPECT/CT revealed intense and focal hyperfixation at the hepatic

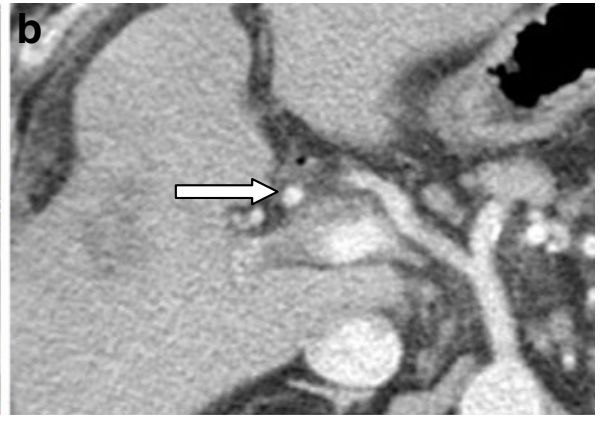

hilum (a, $\longrightarrow$ ) just below the duodenum corresponding to a hepatic artery uptake, as hepatic artery is clearly demonstrated at contrast-enhanced CT (b匚 
Fig. 4 MAA uptake at coil embolization site. A 71-yearold patient with inoperable centrohepatic cholangiocarcinoma. MAA SPECT/CT (a) revealed focal hyperfixation at the coil embolization site after selective injection into the right hepatic artery following coil embolization of the gastroduodenal artery. The injection of $1.95 \mathrm{GBq}$ glass microspheres was performed, vascular uptake not being taken into account. Post-treatment BS (b) showed no microsphere uptake at the coil embolization site. Treatment was well tolerated a

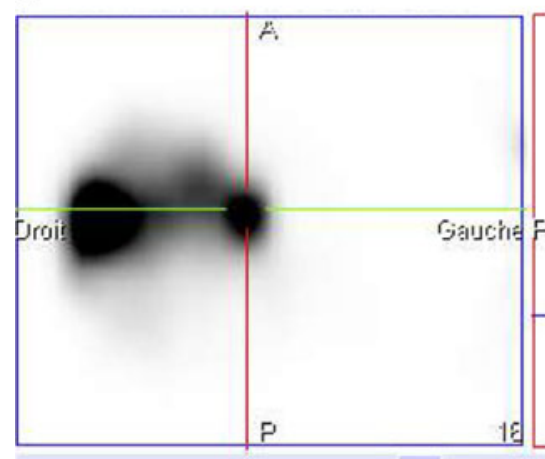

b
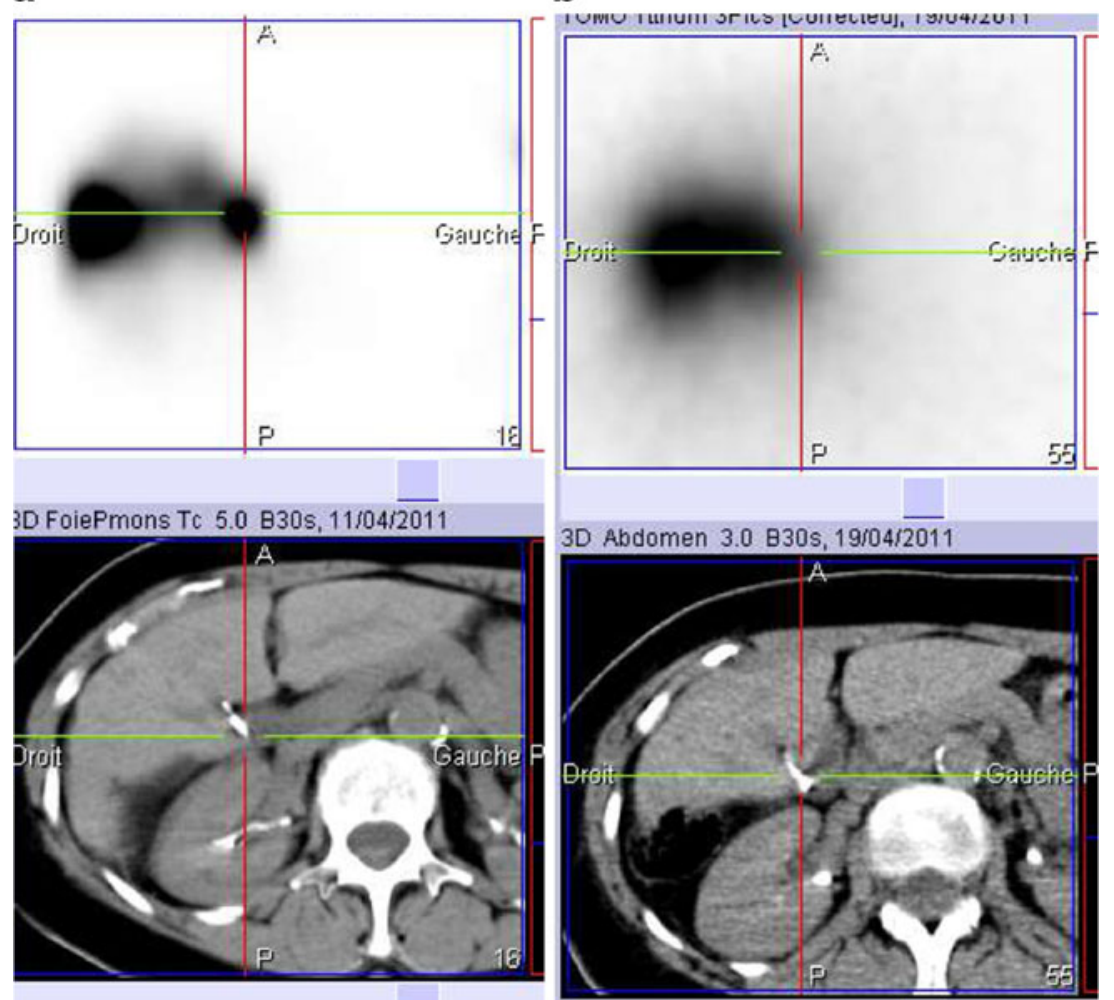

[omo 99mTc [Corrected Recon], 11/ $\overline{04 / 2011}$

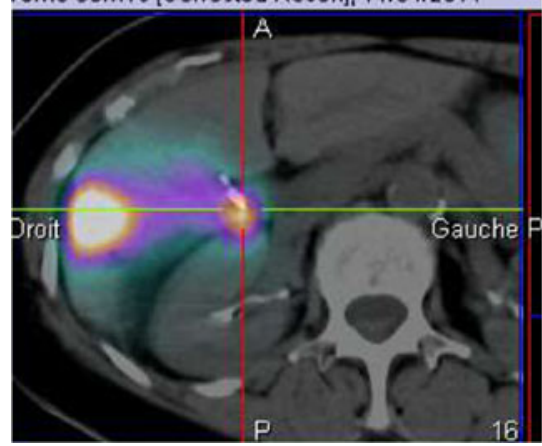

TOMO Yttrium 3Pics [Corrected], $\overline{19 / 04 / 2011}$

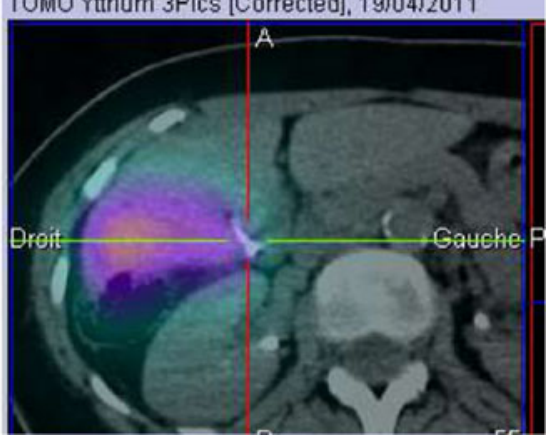

50 times higher number of microspheres for any given injected activity. Given this context, it should be noted that when considering high-volume teams that had treated over 100 patients the risk of ulcer disease after using resin microspheres did not exceed 5.5\% $[13,15,16]$ and less than $1 \%$ after using glass microspheres $[1,2]$.

This ulcer type is often severe, and surgical interventions are required in over $50 \%$ of cases, because of the potential patient resistance to proton pump inhibitors [11], which highlights the need for effectively identifying high-risk patients. With the advent of SPECT/CT hybrid systems, MAA hepatic arterial perfusion scintigraphy may allow detection of digestive shunts, in addition to diagnostic angiography. However, little data are available on the subject [6-8], with only two published studies on SPECT/CT including over 50 patients $[6,7]$.

In the first study by Hamami et al., SPECT/CT allowed detection of 16 cases of uptake suggestive of gastroduodenal shunting, with planar imaging identifying only 4 cases among the 68 scans performed ( $23 \%$ of cases) [6]. In two cases, a new diagnostic angiography failed to reveal any aberrant collateral vessel, and microsphere treatment was therefore contraindicated. In eight cases, a new angiography allowed the radiologists to identify the aberrant artery, with coil embolization performed in six patients, while the remaining two underwent microsphere injection at a more distal site. However, in three cases, hepatic hilar uptake was initially considered to indicate digestive shunting, but a comparison with the injected diagnostic CT scan eventually revealed that the uptake was due to portal vein thrombosis. Recently, the same expert team reported on the results obtained with ${ }^{90} \mathrm{Y}$-microsphere therapy in 108 hepatocellular carcinoma patients, with none of the patients presenting gastric ulcer after therapy [2].

Similarly, in a report published by Ahmadzadehfar et al. involving 90 scans, $42 \%$ extrahepatic uptake was detected 

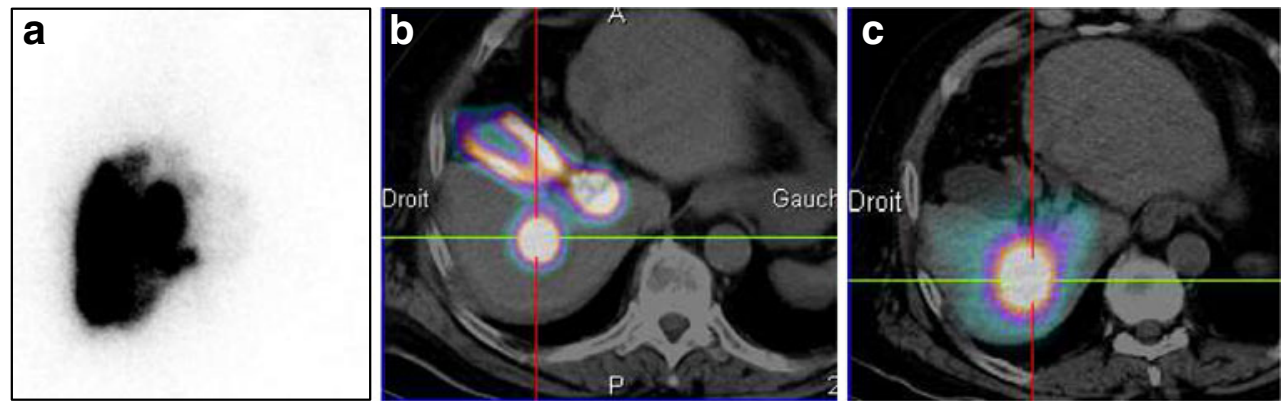

Fig. 5 Gall bladder uptake. A 75-year-old patient with unifocal recurrence of hepatocellular carcinoma at the 5/6 liver segments after chemoembolization. a Planar MAA scintigraphy: heterogeneous liver uptake. b MAA SPECT/CT: high uptake by gall bladder, tumour and hepatic artery. $\mathbf{c}$ Post-treatment BS: absence of hyperfixation of the gall bladder despite the absence of cystic artery stimulation. The patient experienced an episode of subhepatic pain with a vesicular sludge, referred to as alithiasic cholecystitis, which resolved after medical treatment; its relationship to the radioembolization procedure remains unclear because of the absence of gall bladder hyperfixation
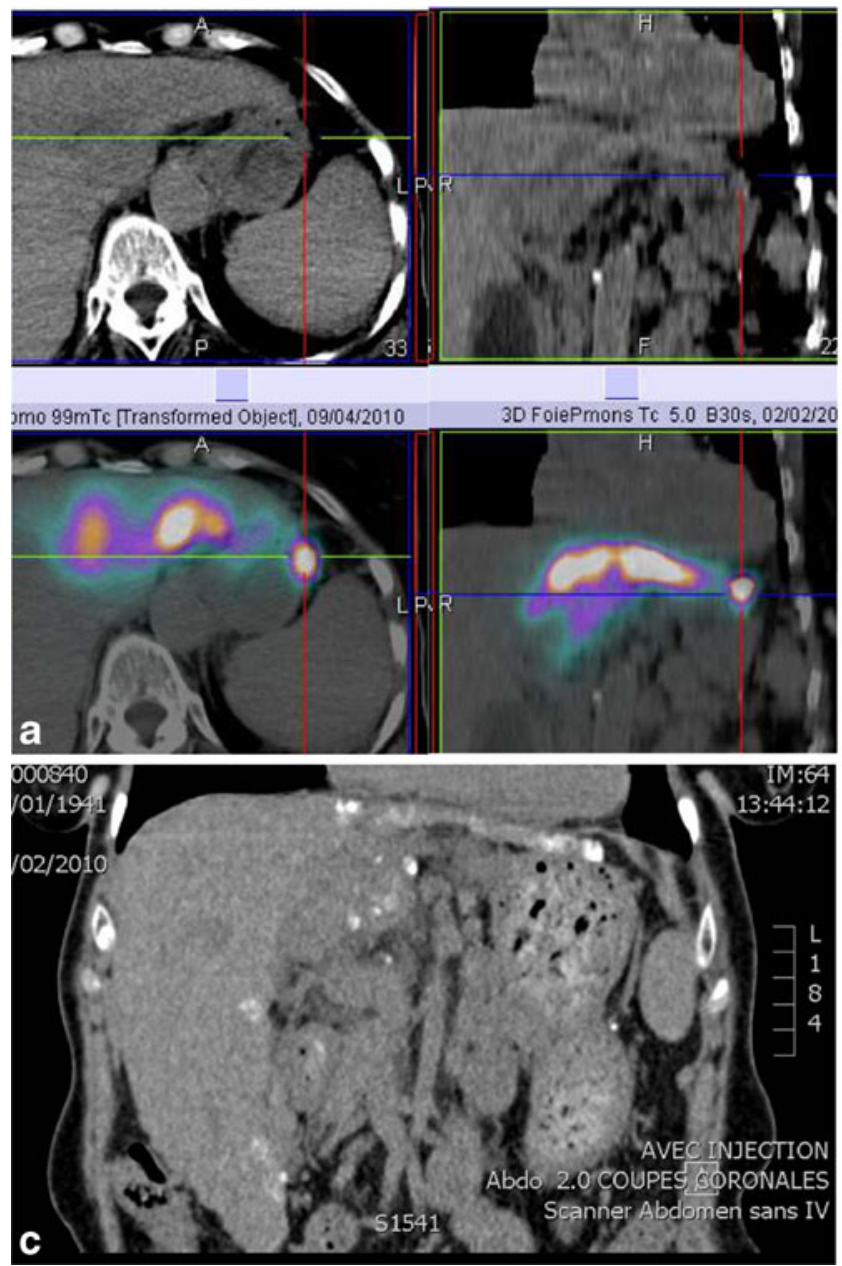

Fig. 6 Coregistration error. A 69-year-old patient with variant hepatic arterial anatomy presenting three hepatic arteries and multifocal hepatocellular carcinoma in segment 1 , infiltrating the left liver lobe, associated with portal vein thrombosis. a MAA SPECT/CT following selective injection into the left hepatic artery: extrahepatic uptake, probably corresponding to the gastric wall. Because of segment 1 involvement and due to the lack of portal vein thrombosis targeting, a second MAA SPECT/CT was performed at the middle hepatic artery level (b) highlighting a coregistration error at the tip of the left liver lobe, with $\mathrm{CT}$ identification of a hepatic tongue, extending along the

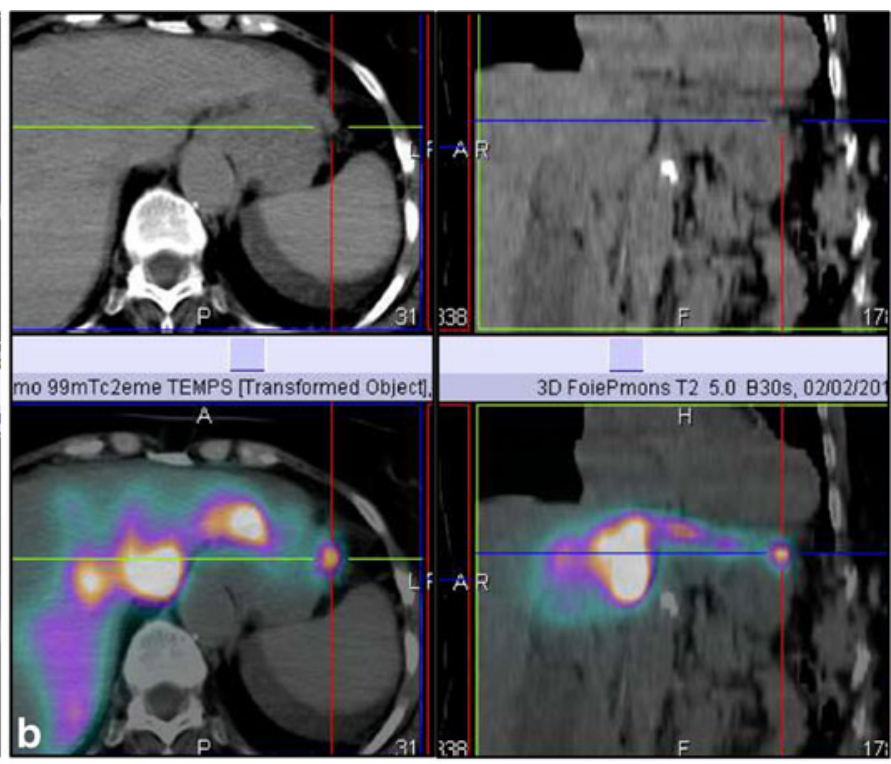

left gastric border. The suspected diagnosis was a small-sized lesion at the tip of the left liver lobe. It was therefore decided to perform firstline treatment of the middle hepatic artery. During the procedure, selective lipiodol arteriography of the left hepatic artery was conducted, with CT lipiodol (c) confirming the presence of a hepatic tongue, along with a nodular lesion taking up lipiodol. Thereafter, 2 months after the first treatment, the patient underwent treatment of the left hepatic artery. The treatment was well tolerated, with the patient being still alive, with good tumour control, 11 months after the first treatment 
using SPECT/CT compared to only $12 \%$ with planar imaging [7]. These results took into account four cases of gastric uptake in relation to free pertechnetate and nine cases of gall bladder uptake. Gastric or duodenal uptake was found 18 times, corresponding to $20 \%$ of cases. For nine patients, rereading the diagnostic angiograms allowed radiologists to detect an aberrant vessel, which was treated by embolization or distal microsphere injections. For two patients, there was no aberrant vessel detected while re-reading diagnostic angiograms, and in both cases, microsphere treatment was considered contraindicated. For four patients, the duodenum was only identified a posteriori as the specific site of uptake following retrospective data analysis. Of these four patients, two developed ulcer disease, one did not undergo treatment because of pulmonary shunting, while the remaining patient underwent coil embolization of the aberrant vessel during therapy. Other uptake sites were found including coil embolization $(n=6)$, intestinal region $(n=9)$, spleen $(n=2)$, abdominal wall $(n=3)$ and diaphragm $(n=1)$.

The recently published study of Dudeck et al. [9] on reangiography following positive MAA SPECT/CT found that among 341 patients gastroduodenal uptake was identified in 33 patients $(9.7 \%)$ using SPECT/CT, while at repeat angiography the source of extrahepatic flow was identified and corrected in 31 patients.

Our findings are in line with published data $[6,7,9]$, demonstrating that SPECT/CT enabled the identification of digestive shunts that were previously overlooked by selective angiography. However, in our series, the incidence rate of digestive shunts that went unnoticed was lower, representing only $4.8 \%$ of cases.

It should be noted that in several cases uptake by vascular structures or, more rarely, misregistration artefacts may render SPECT/CT interpretation difficult (Table 2). The hepatic hilar area is particularly difficult to interpret. Uptake in this area may be of duodenal origin, with a potential impact on treatment decisions, although it may also be due to vascular structures, without therapeutic implications.

In this article, we reported for the first time on MAA uptake by the hepatic artery, observed in $6.6 \%$ of explorations, which had no impact on patient management and was not associated with any complications. These arterial uptakes are likely due to the impaction or aggregation of MAA by arterial microlesions caused by the long and complicated angiography procedures or when arteries are weakened by previous procedures. In fact, among the nine patients with MAA uptake via the hepatic artery, four had previously been administered intra-arterial therapy, consisting of chemoembolization in three cases and ${ }^{131}$ I-lipiodol treatment in one, while one patient had undergone a prior hepatectomy and another had experienced a difficult angiography due to arterial stenosis. Uptake at the coil embolization site may have erroneously led to the diagnosis of duodenal uptake, which was observed in $2.1 \%$ of our explorations, compared to $6.6 \%$ of the explorations reported by Ahmadzadehfar et al.

While uptake by portal vein thrombosis was reported in three cases in the study of Hamami et al., a $6.4 \%$ incidence of portal vein thrombosis uptake was found in our series, approaching $31 \%$ in patients with established portal vein thrombosis. This critical point must be stressed, as portal vein thrombosis is a good indication for radioembolization $[1,2]$.

The presence of free pertechnetate was another source of interpretation error when using SPECT/CT. However, due to the diffuse nature of gastric uptake along with the associated thyroid uptake, it was easy to recognize. Several authors recommend using perchlorate to reduce potential errors [17].

Generally, MAA uptake via the hepatic falciform artery does not result in diagnostic problems except for patients with umbilical hernia. Although coil embolization is usually recommended prior to microsphere injection, the risk of complications appears to be low, since six of our seven patients who received microsphere injections without prior embolization

Table 2 Site and frequency of digestive extrahepatic uptake (expressed as percentage of number of SPECT/CT by study), other than free pertechnetate, reported in the literature

\begin{tabular}{|c|c|c|c|c|c|}
\hline & Current study & Hamami et al. [6] & Ahmadzadehfar et al. [7] & Dudeck et al. [9] & Denecke et al. [8] \\
\hline Number of SPECT/CT & 139 & 68 & 90 & 341 & 28 \\
\hline Focal gastroduodenal uptake (\%) & 3.6 & 23.5 & 24.4 & 9.7 & 31 \\
\hline Portal vein thrombosis uptake $(\%)$ & 6.5 & 4.4 & NR & NR & NR \\
\hline Hepatic artery uptake $(\%)$ & 6.5 & NR & NR & NR & NR \\
\hline Coil uptake (\%) & 2.1 & NR & 6.6 & NR & NR \\
\hline Gall bladder uptake (\%) & 12.2 & 8.8 & 10 & NR & NR \\
\hline Falciform artery uptake (\%) & 5.0 & NR & 3.3 & NR & NR \\
\hline \multirow[t]{2}{*}{ Other uptake $(\%)$} & 0 & NR & $\begin{array}{l}\text { Spleen: } 2.2 \\
\text { Intestine: } 10\end{array}$ & NR & NR \\
\hline & & & Diaphragm: 1.1 & & \\
\hline
\end{tabular}


did not present any complications. In a recent study, a hepatic falciform artery was identified using MAA SPECT/CT a posteriori in 16 patients who did not undergo coil embolization during treatment; only 1 patient complained of abdominal pain for $48 \mathrm{~h}$, without presenting skin lesions [18]. However, identifying a falciform artery and the associated low risk of abdominal pain is important on the one hand to properly inform the patient and on the other to avoid performing multiple and unnecessary endoscopies to detect ulcer disease in patients with unexplained abdominal pain.

Furthermore, SPECT/CT allowed detection of gall bladder uptake, which was not identified using planar imaging, as was the case in $12.2 \%$ of the explorations in the present study.

Although no particular problems were associated with the operational strategy for patients with low-intensity uptake (i.e. inferior to hepatic parenchymal uptake), high-intensity uptake remained a therapeutic challenge with no established consensus.

The therapeutic decision must be made on a case-by-case basis following a precise evaluation of the benefit-risk balance.

The risk of acute cholecystitis is known, with several cases being reported in the literature [19]. However, its frequency appears to be very low given that in a large retrospective multicentre study on 515 patients no case of cholecystitis was reported [20]. Nevertheless, this complication may be treated surgically as was described for two patients presenting acute cholecystitis [19].

In cases where distal positioning of the catheter was not possible, Hamami et al. [6] performed a preventive cholecystectomy in 2 patients, while Ahmadzadehfar et al. [7] carried out coil embolization of the cystic artery in 1 patient and Dudeck et al. [9] in 13, with no complications being observed. In our series, prior to microsphere injection, we performed a catheter stimulation of the cystic artery in two of seven patients with high MAA uptake, for whom this procedure was possible. It is interesting to note that for four patients with intense MAA uptake by the gall bladder no uptake was observed on BS, despite the catheter being in the identical position and the absence of any cystic artery stimulation, which was likely to be due to the instability or vasomotor variability of the cystic artery (Fig. 5). The decision to treat the seven patients with intense gall bladder uptake was taken on a case-by-case basis following a benefit-risk evaluation. It was taken into account that five of these patients required salvage treatment in the setting of very advanced tumour disease, with four presenting portal vein thrombosis. For the two remaining patients, vasomotor variability of the cystic artery was identified in the absence of microspheres on BS. These patients received prophylactic therapy with anti-inflammatory drugs and antibiotics for 14 days following treatment.

Lastly, SPECT/CT interpretation may lead to diagnostic problems if coregistration errors occur due to diaphragm movements. Although poorly documented in the scientific literature, the frequency of these artefact types may be significant when exploring moving anatomical structures. In fact, for the liver, Vogel et al. reported the risk of misregistration errors to be $30-40 \%$ of cases using positron emission tomography (PET)/CT according to respiratory status (expiration breath-hold or free breathing), with the mean error of positioning being $>1 \mathrm{~cm}$ (range 4-16 $\mathrm{mm}$ for free breathing) [21]. Therefore, a coregistration error should be considered systematically before drawing any final conclusion on digestive tract uptake, performing another angiography or contraindicating microsphere therapy definitively.

\section{Conclusion}

This study confirms previously published data suggesting the superiority of SPECT/CT over planar imaging for the identification of digestive, extrahepatic MAA uptake sites, which were found in more than one third of our cases, including gall bladder uptake. MAA SPECT/CT should now be recommended in the preoperative assessment prior to radioembolization in order to better identify the risk of digestive shunts. In cases of gastroduodenal uptake on MAA SPECT/CT, a repeat angiographic assessment is mandatory as the source of gastroduodenal flow can frequently be identified and corrected. However, our series revealed a high frequency of vascular uptake $(20.1 \%)$ at the level of portal vein thrombosis, hepatic artery or coil embolization sites, without any consequences for the therapeutic management of patients. These uptake sites must be recognized by experts, as should coregistration errors, in order to avoid the risk of excluding patients who do not present any contraindications to radioembolization from this effective therapeutic strategy.

Conflicts of interest Etienne Garin is a consultant for Nordion.

\section{References}

1. Salem R, Lewandowski RJ, Mulcahy MF, Riaz A, Ryu RK, Ibrahim S, et al. Radioembolization for hepatocellular carcinoma using yttrium-90 microspheres: a comprehensive report of longterm outcomes. Gastroenterology 2010;138(1):52-64.

2. Hilgard P, Hamami M, Fouly AE, Scherag A, Müller S, Ertle J, et al. Radioembolization with yttrium-90 glass microspheres in hepatocellular carcinoma: European experience on safety and longterm survival. Hepatology 2010;52(5):1741-9.

3. Sangro B, Carpanese L, Cianni R, Golfieri R, Gasparini D, Ezziddin $\mathrm{S}$, on behalf of the European Network on Radioembolization with Yttrium-90 Resin Microspheres (ENRY), et al. Survival after yttrium-90 resin microsphere radioembolization of hepatocellular carcinoma across Barcelona clinic liver cancer stages: a European 
evaluation. Hepatology 2011;54:868-78. doi:10.1002/hep.24451 [Epub ahead of print].

4. Sato KT, Lewandowski RJ, Mulcahy MF, Atassi B, Ryu RK, Gates $\mathrm{V}$, et al. Unresectable chemorefractory liver metastases: radioembolization with $90 \mathrm{Y}$ microspheres-safety, efficacy, and survival. Radiology 2008;247(2):507-15.

5. Hendlisz A, Van den Eynde M, Peeters M, Maleux G, Lambert B, Vannoote J, et al. Phase III trial comparing protracted intravenous fluorouracil infusion alone or with yttrium-90 resin microspheres radioembolization for liver-limited metastatic colorectal cancer refractory to standard chemotherapy. J Clin Oncol 2010;28(23): 3687-94.

6. Hamami ME, Poeppel TD, Müller S, Heusner T, Bockisch A, Hilgard $\mathrm{P}$, et al. SPECT/CT with 99mTc-MAA in radioembolization with $90 \mathrm{Y}$ microspheres in patients with hepatocellular cancer. J Nucl Med 2009;50(5):688-92.

7. Ahmadzadehfar H, Sabet A, Biermann K, Muckle M, Brockmann $\mathrm{H}$, Kuhl C, et al. The significance of 99mTc-MAA SPECT/CT liver perfusion imaging in treatment planning for $90 \mathrm{Y}$-microsphere selective internal radiation treatment. J Nucl Med 2010;51(8): 1206-12.

8. Denecke T, Rühl R, Hildebrandt B, Stelter L, Grieser C, Stiepani $\mathrm{H}$, et al. Planning transarterial radioembolization of colorectal liver metastases with yttrium 90 microspheres: evaluation of a sequential diagnostic approach using radiologic and nuclear medicine imaging techniques. Eur Radiol 2008;18(5):892-902.

9. Dudeck O, Wilhelmsen S, Ulrich G, Löwenthal D, Pech M, Amthauer H, et al. (2011) Effectiveness of repeat angiographic assessment in patients designated for radioembolization using yttrium-90 microspheres with initial extrahepatic accumulation of technetium- $99 \mathrm{~m}$ macroaggregated albumin: a single center's experience. Cardiovasc Intervent Radiol [Epub ahead of print].

10. Garin E, Rolland Y, Boucher E, Ardisson V, Laffont S, Boudjema $\mathrm{K}$, et al. First experience of hepatic radioembolization using microspheres labelled with yttrium-90 (TheraSphere ${ }^{\circledR}$ ): practical aspects concerning its implementation. Eur J Nucl Med Mol Imaging 2010;37:453-61.

11. Naymagon S, Warner RRP, Patel K, Harpaz N, Machac J, Weintraub $\mathrm{JL}$, et al. Gastroduodenal ulceration associated with radioembolization for the treatment of hepatic tumors: an institutional experience and review of the literature. Dig Dis Sci 2010;55:2450-8.
12. Dancey JE, Shepherd FA, Paul K, Sniderman KW, Houle S, Gabrys $\mathrm{J}$, et al. Treatment of nonresectable hepatocellular carcinoma with intrahepatic 90Y-microspheres. J Nucl Med 2000;41:1673-81.

13. Stubbs RS, Cannan RJ, Mitchell AW. Selective internal radiation therapy with $90 y$ ttrium microspheres for extensive colorectal liver metastases. J Gastrointest Surg 2001;5(3):294-302.

14. Lim L, Gibbs P, Yip D, Shapiro JD, Dowling R, Smith D, et al. A prospective evaluation of treatment with Selective Internal Radiation Therapy (SIR-spheres) in patients with unresectable liver metastases from colorectal cancer previously treated with 5 -FU based chemotherapy. BMC Cancer 2005;5:132.

15. Kennedy AS, McNeillie P, Dezarn WA, Nutting C, Sangro B, Wertman D, et al. Treatment parameters and outcome in 680 treatments of internal radiation with resin 90Y-microspheres for unresectable hepatic tumors. Int J Radiat Oncol Biol Phys 2009;74 (5):1494-500.

16. Konda A, Savin MA, Cappell MS, Duffy MC. Radiation microsphere-induced GI ulcers after selective internal radiation therapy for hepatic tumors: an underrecognized clinical entity. Gastrointest Endosc 2009;70(3):561-7.

17. Sabet A, Ahmadzadehfar H, Muckle M, Haslerud T, Wilhelm K, Biersack HJ, et al. Significance of oral administration of sodium perchlorate in planning liver-directed radioembolization. J Nucl Med 2011;52(7):1063-7.

18. Ahmadzadehfar H, Möhlenbruch M, Sabet A, Meyer C, Muckle $\mathrm{M}$, Haslerud T, et al. Is prophylactic embolization of the hepatic falciform artery needed before radioembolization in patients with 99mTc-MAA accumulation in the anterior abdominal wall? Eur J Nucl Med Mol Imaging 2011;38(8):1477-84.

19. Carr BI. Hepatic arterial 90 Yttrium glass microspheres (Therasphere) for unresectable hepatocellular carcinoma: interim safety and survival data on 65 patients. Liver Transpl 2004;10(2 Suppl 1):S107-10.

20. Kennedy AS, McNeillie P, Dezarn WA, Nutting C, Sangro B, Wertman D, et al. Treatment parameters and outcome in 680 treatments of internal radiation with resin 90Y-microspheres for unresectable hepatic tumors. Int J Radiat Oncol Biol Phys 2009;74 (5):1494-500.

21. Vogel WV, van Dalen JA, Wiering B, Huisman H, Corstens FH, Ruers TJ, et al. Evaluation of image registration in PET/CT of the liver and recommendations for optimized imaging. J Nucl Med 2007;48(6):910-9. 